\title{
Imperfect CSI and Improper Gaussian Noise Effects on SSK: Optimal Detection and Error Analysis
}

\author{
Malek M. Alsmadi ${ }^{\mathrm{a}}$, Ayse E. Canbilen ${ }^{\mathrm{a}, \mathrm{c}}$, Salama S. Ikki ${ }^{\mathrm{a}}$, Ertugrul Basar ${ }^{\mathrm{b}}$, Seyfettin S. Gultekin ${ }^{\mathrm{c}}$, Ibrahim Develi ${ }^{\mathrm{d}}$ \\ ${ }^{a}$ Department of Electrical Engineering, Faculty of Engineering, Lakehead University, Thunder Bay, Ontario, Canada. \\ E-mail: \{malsamdi, acanbile, sikki\}@ lakeheadu.ca \\ ${ }^{\mathrm{b}}$ CoreLab, Department of Electrical and Electronics Engineering, Koç University, Sariyer 34450, Istanbul, Turkey. \\ E-mail: ebasar@ku.edu.tr \\ ${ }^{\mathrm{c}}$ Department of Electrical and Electronics Engineering, Faculty of Engineering, Selcuk University, Konya, Turkey. \\ E-mail: \{ayseelif, sgultekin\}@ selcuk.edu.tr \\ ${ }^{\mathrm{d}}$ Department of Electrical and Electronics Engineering, Faculty of Engineering, Erciyes University, Kayseri, Turkey. \\ E-mail: develi@erciyes.edu.tr
}

\begin{abstract}
Space shift keying (SSK) has many advantages through its unique transmission manner as compared to other multiple-input multiple-output (MIMO) techniques. Nevertheless, the practicality of SSK in the presence of real-time imperfections such as channel estimation errors and hardware impairments (HWIs) is still an open research problem. On the other hand, the effects of HWIs are assumed as zeromean circularly-symmetric complex Gaussian random variable (RV) in the literature. However, this model does not reflect the asymmetric characteristics of different HWIs. Therefore, the aim of this paper is to shed light on the joint effect of improper Gaussian noise (IGN) and imperfect channel state information (ICSI) on the performance of SSK receiver. Particularly, an optimal maximum likelihood (ML) detector is designed, and pairwise error probability (PEP) expression is derived. Additionally, an exact closed-form Cramer-Rao bound expression is calculated for evaluating the channel estimation accuracy under the effect of IGN. The results obtained by using computer simulations prove that the proposed optimal detector is superior to the traditional ML detector in the presence of IGN and ICSI.
\end{abstract}

Index Terms-Improper Gaussian noise, imperfect channel state information, multiple input multiple output, space shift keying, optimal receiver, error performance analysis.

\section{INTRODUCTION}

The accelerated developments in the mobile communication systems technology are moving the world toward a fully connected network with new challenges, including the inevitable trade-off between spectral efficiency and energy efficiency [1]. One of the key solutions to deal with this problem is utilizing multiple-input multiple-output (MIMO) techniques, such as space shift keying (SSK) [2]. The fundamental idea of SSK was originally proposed in [3]. In SSK, a single transmitter (Tx) antenna among the set of existing Tx antennas is activated during each time instant and the index of this active antenna is used to convey information [4]. It means that the transmitted signal does not contain a constellation symbol, and the receiver ( $\mathrm{Rx})$ determines only the active Tx antenna index for each particular time slot. Activating a single $\mathrm{Tx}$ antenna at each time instant eliminates interchannel interference, reduces the system complexity, avoids the need for the inter-antenna synchronization, and minimizes the effects of channel estimation errors [5].
Several schemes based on the unique manner of SSK have been investigated in the literature. For instance, an accurate framework for evaluating the performance of SSK over Rayleigh fading channels in the presence of imperfect channel state information (ICSI) was presented in [6], and a simple asymptotic approximation for the error probability was also defined. The joint impact of ICSI and co-channel interference (CCI) on the performance of SSK MIMO systems was investigated in [7]. Most recently, a general analytical SSK MIMO system scheme, which considers the effect of hardware impairments (HWIs) and CCI, was introduced in [8], and the deterioration effect of HWIs on SSK was proven.

On the other hand, modeling the effect of HWIs as a zero-mean circularly-symmetric complex Gaussian random variable (RV) fails to reflect the asymmetric characteristics of the HWIs. Therefore, it is necessary to consider the effect of HWIs as improper Gaussian noise (IGN) to capture their characteristics [9]. A complex RV is called proper RV if its real and imaginary parts are circularly symmetric (uncorrelated and have the same variance). Otherwise, it is called improper RV [10]. Moreover, it is shown that exploiting the improperness of the Gaussian noise using ML detector leads to decreasing of the error probability when detecting the binary signals [11].

All the above-mentioned works motivated the authors to study the joint effect of IGN and ICSI on SSK modulation by accurately modifying the traditional ML detector. In this paper, the general SSK model has been defined under the assumption of independent IGN and ICSI at each SSK Rx. Specifically, we have designed an optimal ML detector, solved the pairwise error probability (PEP) problem and derived an exact closed-form Cramer-Rao lower bound (CRLB) expression. Computer simulation results prove the superiority of the proposed optimal design compared to the traditional ML detector. This work might be useful for future researches that consider HWIs by $i$ ) improving our understanding of IGN effect on SSK MIMO systems, ii) showing the effect of IGN on channel estimation accuracy.

Organization: SSK MIMO system model is described in Section II. Optimal and sub-optimal ML detectors in the presence of the joint effect of IGN and ICSI are presented in 
Section III. Next, error performance analyses for the proposed detectors are given in Section IV. The numerical analysis and results are shown in Section V. Finally, the work is concluded in Section VI.

Notations: The scalar values are denoted by italic symbols. Vectors and matrices are denoted by bold lower and bold upper italic symbols, respectively. Vector transpose is represented by $(\cdot)^{T}$, and the Frobenius norm of a vector/matrix is represented by $\|\cdot\|^{2} \cdot \mathbb{E}\{\cdot\}$ is the expectation operator, and $\Re\{\cdot\}$ denotes the real part of a complex RV. $\mathcal{C N}\left(\mu, \sigma^{2}\right)$ represents the complex-valued Gaussian distribution with mean $\mu$ and variance $\sigma^{2} . Q(x)=\frac{1}{\sqrt{2 \pi}} \int_{x}^{\infty} \exp \left(\frac{-u^{2}}{2}\right) d u$.

\section{SYSTEM AND CHANNEL MODELS}

\section{A. SSK Transceiver}

SSK transceiver model is basically defined as an $N_{t} \times N_{r}$ MIMO system, where $N_{t}$ and $N_{r}$ are the numbers of Tx and $\mathrm{Rx}$ antennas, respectively. First of all, Tx maps blocks of equiprobable $m=\log _{2} N_{t}$ incoming bits into the index of a single Tx antenna, which is activated for data transmission while all other Tx antennas are kept silent [6]. Then, the signal is conveyed over an $N_{r} \times N_{t}$ complex wireless channel $\boldsymbol{H}$. Each element of $\boldsymbol{H}$, shown by $h_{i, j}$, stands for a complex fading channel gain between the $i^{\text {th }} \mathrm{Rx}$ antenna and $j^{\text {th }}$ Tx antenna $\left(i \in\left\{1, \ldots, N_{r}\right\}, j \in\left\{1, \ldots, N_{t}\right\}\right)$. Here, $h_{i, j}$ is assumed as independent and identically-distributed complex Gaussian RVs with zero-mean and unit variance. Considering that only one Tx antenna is activated, the output of the channel can be given by

$$
\boldsymbol{y}=\sqrt{E} \boldsymbol{h}_{j}+\boldsymbol{n},
$$

where $\boldsymbol{h}_{j}$ is the $j^{\text {th }}$ column of the channel coefficients matrix $\boldsymbol{H}$, i.e., $\boldsymbol{h}_{j}=\left[h_{1, j}, \ldots, h_{N_{r}, j}\right]^{T}$, while $E$ is the transmitted signal energy. In addition to this, $\boldsymbol{n}=\left[n_{1}, \ldots, n_{N_{r}}\right]^{T}$ is the IGN vector that has independent improper Gaussian RVs with distribution of $\mathcal{C N}(0,1)$. Note that there are two special cases for improper RV; Case 1: identical correlated RV, when the real and the imaginary parts are correlated and have equal variances, Case 2: non-identical uncorrelated RV, when the real and the imaginary parts are not correlated but have different variances. However, in order to generalize the results, the general case in which the real and imaginary parts of $\boldsymbol{n}$ are non-identical and correlated Gaussian RVs with variances of $\sigma_{I}^{2}$ and $\sigma_{Q}^{2}$, respectively, are considered in this study. (Note that, $\sigma_{n}^{2}=\sigma_{I}^{2}+\sigma_{Q}^{2}$ )

In the case of ICSI, Rx needs to estimate channel coefficients. The estimated channel coefficients at the Rx side can be characterized as follows [12]

$$
\boldsymbol{h}_{j}=\hat{\boldsymbol{h}}_{j}+\boldsymbol{e}_{j}
$$

where $\hat{\boldsymbol{h}}_{j}$ is the $j^{\text {th }}$ column of the estimated channel coefficients matrix, $\hat{\boldsymbol{h}}_{j}=\left[\hat{h}_{1, j}, \ldots, \hat{h}_{N_{r}, j}\right]^{T}$, and $\boldsymbol{e}_{j}$ is the $j^{\text {th }}$ column of the channel estimation error matrix, $\boldsymbol{e}_{j}=$ $\left[e_{1, j}, \ldots, e_{N_{r}, j}\right]^{T}$. In addition, $e_{i, j} \sim \mathcal{C N}\left(0, \sigma_{e}^{2}\right)$ is the channel estimation error between the $i^{t h} \mathrm{Rx}$ antenna and $j^{\text {th }} \mathrm{Tx}$ antenna, $e_{i, j}$ is also supposed to have improper Gaussian RVs with $\sigma_{e_{I}}^{2}$ real part variance and $\sigma_{e_{Q}}^{2}$ imaginary part variance. Note that the variance of $e_{i, j}$ includes the information of the channel estimation quality [6].

\section{B. Cramer-Rao Lower Bound (CRLB)}

The CRLB provides a lower bound for the variance of an unbiased estimator of a parameter. In this section, an exact expression is derived for CRLB of the channel estimation in the presence of IGN at the Rx side. This expression can be used as a benchmark to predict and evaluate any estimator performance in the presence of IGN.

Lemma: The exact Fisher information matrix (FIM) at each SSK Rx can be given as [13]

$$
I_{(\theta)}(\theta)=\left[\begin{array}{cc}
I_{\left(h^{I}\right)} & I_{\left(h^{I}, h^{Q}\right)} \\
I_{\left(h^{I}, h^{Q}\right)} & I_{\left(h^{Q}\right)}
\end{array}\right],
$$

where $\theta=\left[h^{I}, h^{Q}\right]$. This is equal to

$$
I_{(\theta)}(\theta)=\left[\begin{array}{cc}
\frac{N_{p} E}{\left(1-\rho^{2}\right) \sigma_{I}^{2}} & -\frac{N_{p} \rho E}{\left(1-\rho^{2}\right) \sigma_{I} \sigma_{Q}} \\
-\frac{N_{p} \rho E}{\left(1-\rho^{2}\right) \sigma_{I} \sigma_{Q}} & \frac{N_{p} E}{\left(1-\rho^{2}\right) \sigma_{Q}^{2}}
\end{array}\right],
$$

where $N_{P}$ is the number of training pilots. CRLB matrix can be obtained by finding $\left(I_{(\theta)}(\theta)\right)^{-1}$ as

$$
\mathrm{CRLB}=\left[\begin{array}{cc}
\frac{\sigma_{I}^{2}}{N_{p} E} & \frac{\sigma_{I} \sigma_{Q} \rho}{N_{p} E} \\
\frac{\sigma_{I} \sigma_{Q} \rho}{N_{p} E} & \frac{\sigma_{Q}^{2}}{N_{p} E}
\end{array}\right] .
$$

Proof: See the Appendix.

\section{Maximum Likelihood (ML) Detection}

In this section, optimal and sub-optimal ML detectors are presented for the SSK MIMO wireless communication system which is under the effects of both ICSI and IGN.

\section{A. Optimal ML Detection}

From (1) and (2), the received signal $\boldsymbol{y}$ can be expressed as

$$
\boldsymbol{y}=\sqrt{E} \hat{\boldsymbol{h}}_{j}+\underbrace{\sqrt{E} \boldsymbol{e}_{j}+\boldsymbol{n}}_{\boldsymbol{z}} .
$$

From (6), it clear that $z$ is a zero-mean IGN and the variances of its real part $\boldsymbol{z}^{I}$ and imaginary part $\boldsymbol{z}^{Q}$ are $\delta_{I}^{2}=E \sigma_{e_{I}}^{2}+$ $\sigma_{I}^{2}$ and $\delta_{Q}^{2}=E \sigma_{e_{Q}}^{2}+\sigma_{Q}^{2}$, respectively. Based on that, the conditional joint probability density function (PDF) of the real part, $\boldsymbol{y}^{I}$, and the imaginary part, $\boldsymbol{y}^{Q}$, of the received signal can be given as

$$
\begin{aligned}
f_{\boldsymbol{y}^{I}, \boldsymbol{y}^{Q}}\left(\boldsymbol{y}^{I}, \boldsymbol{y}^{Q} \mid \boldsymbol{h}_{j}\right)=\left(\frac{1}{2 \pi \delta_{I} \delta_{Q} \sqrt{1-\rho^{2}}}\right)^{N_{r}} \exp \left(\frac{-1}{2\left(1-\rho^{2}\right)}\right. \\
{\left[\frac{\left\|\boldsymbol{y}^{I}-\sqrt{E} \hat{\boldsymbol{h}}_{j}^{I}\right\|^{2}}{\delta_{I}^{2}}+\frac{\left\|\boldsymbol{y}^{Q}-\sqrt{E} \hat{\boldsymbol{h}}_{j}^{Q}\right\|^{2}}{\delta_{Q}^{2}}\right.} \\
\left.\left.-\frac{2 \rho\left(\boldsymbol{y}^{I}-\sqrt{E} \hat{\boldsymbol{h}}_{j}^{I}\right)^{T}\left(\boldsymbol{y}^{Q}-\sqrt{E} \hat{\boldsymbol{h}}_{j}^{Q}\right)}{\delta_{I} \delta_{Q}}\right]\right),
\end{aligned}
$$

where $\hat{\boldsymbol{h}}_{j}^{I}$ and $\hat{\boldsymbol{h}}_{j}^{Q}$ are the real and imaginary parts of $\hat{\boldsymbol{h}}_{j}$, respectively, and $\rho=\mathbb{E}\left\{\boldsymbol{z}^{I} \boldsymbol{z}^{Q}\right\} /\left(\delta_{I} \delta_{Q}\right)$. Assuming that the channel inputs are equiprobable, the optimal ML detector is defined based on maximizing the joint PDF given in (7) as follows 


$$
\begin{aligned}
& \hat{j}=\arg \max _{j}\left\{( \frac { 1 } { 2 \pi \delta _ { I } \delta _ { Q } \sqrt { 1 - \rho ^ { 2 } } } ) ^ { N _ { r } } \operatorname { e x p } \left(-\frac{1}{2\left(1-\rho^{2}\right)}\right.\right. \\
& {\left[\frac{\left\|\boldsymbol{y}^{I}-\sqrt{E} \hat{\boldsymbol{h}}_{j}^{I}\right\|^{2}}{\delta_{I}^{2}}+\frac{\left\|\boldsymbol{y}^{Q}-\sqrt{E} \hat{\boldsymbol{h}}_{j}^{Q}\right\|^{2}}{\delta_{Q}^{2}}\right.} \\
&\left.\left.\left.-\frac{2 \rho\left(\boldsymbol{y}^{I}-\sqrt{E} \hat{\boldsymbol{h}}_{j}^{I}\right)^{T}\left(\boldsymbol{y}^{Q}-\sqrt{E} \hat{\boldsymbol{h}}_{j}^{Q}\right)}{\delta_{I} \delta_{Q}}\right]\right)\right\} .
\end{aligned}
$$

This expression can be written in a simpler from as

$$
\begin{aligned}
\hat{j}=\arg \min _{j}\left\{\frac{\left\|\boldsymbol{y}^{I}-\sqrt{E} \hat{\boldsymbol{h}}_{j}^{I}\right\|^{2}}{\delta_{I}^{2}}+\frac{\left\|\boldsymbol{y}^{Q}-\sqrt{E} \hat{\boldsymbol{h}}_{j}^{Q}\right\|^{2}}{\delta_{Q}^{2}}\right. \\
\left.-\frac{2 \rho\left(\boldsymbol{y}^{I}-\sqrt{E} \hat{\boldsymbol{h}}_{j}^{I}\right)^{T}\left(\boldsymbol{y}^{Q}-\sqrt{E} \hat{\boldsymbol{h}}_{j}^{Q}\right)}{\delta_{I} \delta_{Q}}\right\} .
\end{aligned}
$$

\section{B. Sub-Optimal ML Detection}

The sub-optimal ML detector is defined by using the traditional ML detection method which ignores the improper characteristics of the noise. In this case, the sub-optimal ML detector for the SSK MIMO system is given as

$$
\hat{j}=\arg \max _{j}\left\{\frac{1}{\left(\sqrt{2 \pi \delta_{n}^{2}}\right)^{N_{r}}} \exp \left(-\frac{1}{2}\left\|\boldsymbol{y}-\sqrt{E} \hat{\boldsymbol{h}}_{j}\right\|^{2}\right)\right\} .
$$

Maximizing (10) is also equal to

$$
\hat{j}=\arg \min _{j}\left\{\left\|\boldsymbol{y}-\sqrt{E} \hat{\boldsymbol{h}}_{j}\right\|^{2}\right\} .
$$

\section{Error Performance Analysis}

Given the decision rules in (9) and (11), an error occurs if the antenna $j$ is activated at the Tx, but the Rx erroneously decides antenna $k\left(k \in\left\{1, \ldots, N_{t}\right\}, j \neq k\right)$, and vice versa $\left(\operatorname{Pr}\left(h_{j \mid k}\right)=\operatorname{Pr}\left(h_{k \mid j}\right)\right)$. This is valid for any pair of $h_{j}$ and $h_{k}$. Hence, the error probability for this pair of $h, P_{e}$, can be calculated as

$$
P_{e}=\frac{1}{2} \mathrm{P}_{r}\left(h_{j \mid j}<h_{k \mid j}\right)+\frac{1}{2} \mathrm{P}_{r}\left(h_{k \mid k}<h_{j \mid k}\right) .
$$

In the following sub-sections, first the PEP expression for optimal and non-optimal detectors are derived, and then the bit error rate (BER) is expressed for both of them.

\section{A. PEP of Optimal ML Detector}

Noting that $\operatorname{Pr}\left(h_{j \mid k}\right)=\operatorname{Pr}\left(h_{k \mid j}\right)$ and considering (9), PEP of the optimal ML Rx can be written as follows (utilizing the case that $\boldsymbol{h}_{j}$ is activated at the Tx, but the Rx erroneously decides $\boldsymbol{h}_{k}$ )

$$
\begin{aligned}
\mathrm{P}\left(\boldsymbol{h}_{j} \rightarrow \boldsymbol{h}_{k}\right)_{\mathrm{opt}}=\operatorname{Pr} & \left(\frac{\left\|\boldsymbol{y}^{I}-\sqrt{E} \hat{\boldsymbol{h}}_{j}^{I}\right\|^{2}}{\delta_{I}^{2}}+\frac{\left\|\boldsymbol{y}^{Q}-\sqrt{E} \hat{\boldsymbol{h}}_{j}^{Q}\right\|^{2}}{\delta_{Q}^{2}}\right. \\
& -\frac{2 \rho\left(\boldsymbol{y}^{I}-\sqrt{E} \hat{\boldsymbol{h}}_{j}^{I}\right)^{T}\left(\boldsymbol{y}^{Q}-\sqrt{E} \hat{\boldsymbol{h}}_{j}^{Q}\right)}{\delta_{I} \delta_{Q}}> \\
& \frac{\left\|\boldsymbol{y}^{I}-\sqrt{E} \hat{\boldsymbol{h}}_{k}^{I}\right\|^{2}}{\delta_{I}^{2}}+\frac{\left\|\boldsymbol{y}^{Q}-\sqrt{E} \hat{\boldsymbol{h}}_{k}^{Q}\right\|^{2}}{\delta_{Q}^{2}} \\
& \left.-\frac{2 \rho\left(\boldsymbol{y}^{I}-\sqrt{E} \hat{\boldsymbol{h}}_{k}^{I}\right)^{T}\left(\boldsymbol{y}^{Q}-\sqrt{E} \hat{\boldsymbol{h}}_{k}^{Q}\right)}{\delta_{I} \delta_{Q}}\right) .
\end{aligned}
$$

After some mathematical operations, (13) can be given as

$$
\begin{aligned}
\mathrm{P}\left(\boldsymbol{h}_{j} \rightarrow \boldsymbol{h}_{k}\right)_{\mathrm{opt}}=\operatorname{Pr}\{N & >\frac{E\left\|\hat{\boldsymbol{h}}_{j}^{I}-\hat{\boldsymbol{h}}_{k}^{I}\right\|^{2}}{\delta_{I}^{2}}+\frac{E\left\|\hat{\boldsymbol{h}}_{j}^{Q}-\hat{\boldsymbol{h}}_{k}^{Q}\right\|^{2}}{\delta_{Q}^{2}} \\
& \left.-\frac{2 \rho E\left(\hat{\boldsymbol{h}}_{j}^{I}-\hat{\boldsymbol{h}}_{k}^{I}\right)^{T}\left(\hat{\boldsymbol{h}}_{j}^{Q}-\hat{\boldsymbol{h}}_{k}^{Q}\right)}{\delta_{I} \delta_{Q}}\right\} .
\end{aligned}
$$

Here, $N$ is equal to

$$
\begin{aligned}
N= & \frac{2 \rho \sqrt{E}\left[\left(\hat{\boldsymbol{h}}_{j}^{I}-\hat{\boldsymbol{h}}_{k}^{I}\right)^{T} \boldsymbol{z}^{Q}+\left(\hat{\boldsymbol{h}}_{j}^{Q}-\hat{\boldsymbol{h}}_{k}^{Q}\right)^{T} \boldsymbol{z}^{I}\right]}{\delta_{I} \delta_{Q}} \\
& -2 \sqrt{E}\left[\frac{\left(\hat{\boldsymbol{h}}_{j}^{I}-\hat{\boldsymbol{h}}_{k}^{I}\right)^{T} \boldsymbol{z}^{I}}{\delta_{I}^{2}}-\frac{\left(\hat{\boldsymbol{h}}_{j}^{Q}-\hat{\boldsymbol{h}}_{k}^{Q}\right)^{T} \boldsymbol{z}^{Q}}{\delta_{Q}^{2}}\right] .
\end{aligned}
$$

It is noteworthy that $N$ is a Gaussian RV with zero-mean, and its variance is calculated as follows

$$
\begin{aligned}
\sigma_{N}^{2}=4 E\left(1-\rho^{2}\right) & {\left[\frac{\left\|\hat{\boldsymbol{h}}_{j}^{I}-\hat{\boldsymbol{h}}_{k}^{I}\right\|^{2}}{\delta_{I}^{2}}+\frac{\left\|\hat{\boldsymbol{h}}_{j}^{Q}-\hat{\boldsymbol{h}}_{k}^{Q}\right\|^{2}}{\delta_{Q}^{2}}\right.} \\
& \left.-\frac{2 \rho\left[\left(\hat{\boldsymbol{h}}_{j}^{I}-\hat{\boldsymbol{h}}_{k}^{I}\right)^{T}\left(\hat{\boldsymbol{h}}_{j}^{Q}-\hat{\boldsymbol{h}}_{k}^{Q}\right)\right]}{\delta_{I} \delta_{Q}}\right] .
\end{aligned}
$$

Hence, utilizing (14) and (16), $\mathrm{P}\left(\boldsymbol{h}_{j} \rightarrow \boldsymbol{h}_{k}\right)$ can also be written by using the well-known $Q$-function as given in (17) at the top of the next page, where $\lambda_{\text {opt }}=$ $\left(\frac{\left\|\hat{\boldsymbol{h}}_{j}^{I}-\hat{\boldsymbol{h}}_{k}^{I}\right\|^{2}}{\delta_{I}^{2}}+\frac{\left\|\hat{\boldsymbol{h}}_{j}^{Q}-\hat{\boldsymbol{h}}_{k}^{Q}\right\|^{2}}{\delta_{Q}^{2}}-\frac{2 \rho\left(\hat{\boldsymbol{h}}_{j}^{I}-\hat{\boldsymbol{h}}_{k}^{I}\right)^{T}\left(\hat{\boldsymbol{h}}_{j}^{Q}-\hat{\boldsymbol{h}}_{k}^{Q}\right)}{\delta_{I} \delta_{Q}}\right)$.

The accuracy of the proposed optimal ML detector can be proved by assuming perfect CSI and proper gaussian noise (PGN), i.e., $\delta_{I}^{2}=\delta_{Q}^{2}=\sigma_{n}^{2} / 2$ and $\rho=0$. In this case, the error probability in (17) can be simplified to well-known detection formula as [6]

$$
\mathrm{P}\left(\boldsymbol{h}_{j} \rightarrow \boldsymbol{h}_{k}\right)=Q\left(\sqrt{\frac{E\left\|\boldsymbol{h}_{j}-\boldsymbol{h}_{k}\right\|^{2}}{2 \sigma_{n}^{2}}}\right) .
$$

In order to find the average PEP (APEP) for the optimal ML detector, we need to find the expected value of the expression with the $Q$-function given in (17). Since it is not easy to find the PDF of $\lambda_{\text {opt }}$, which is necessary to calculate this expectation, the moment generating function (MGF) of $\lambda_{\text {opt }}$ is utilized. Noting that $\lambda_{\text {opt }}$ is a quadratic form of Gaussian RVs, the required MGF is equal to

$$
M_{\lambda_{\mathrm{opt}}}(t)=\left(\frac{1}{\sqrt{1-2 \psi_{1} t}} \times \frac{1}{\sqrt{1-2 \psi_{2} t}}\right)^{N_{r}},
$$

where $\psi_{1}$ and $\psi_{2}$ are calculated from

$$
\psi_{1,2}=\frac{1}{2 \delta_{I}^{2}}+\frac{1}{2 \delta_{Q}^{2}} \pm \sqrt{\left(\frac{1}{2 \delta_{I}^{2}}+\frac{1}{2 \delta_{Q}^{2}}\right)^{2}-\frac{\left(1-\rho^{2}\right)}{\delta_{I}^{2} \delta_{Q}^{2}}} .
$$

Now, an exact closed-form expression of the APEP can be given by using (17) and (19) as follows

$$
\bar{P}\left(\boldsymbol{h}_{j} \rightarrow \boldsymbol{h}_{k}\right)_{\mathrm{opt}}=\frac{1}{\pi} \int_{0}^{\frac{\pi}{2}} M_{\lambda_{\text {opt }}}\left(-\frac{E}{8\left(1-\rho^{2}\right) \sin ^{2} \theta}\right) d \theta .
$$

The integration in (21) can be calculated with simple numerical integration techniques. Furthermore, (21) can be simply upper bounded by

$$
\overline{\mathrm{P}}\left(\boldsymbol{h}_{j} \rightarrow \boldsymbol{h}_{k}\right)_{\mathrm{opt}} \leq \frac{1}{2} M_{\lambda_{\text {opt }}}\left(-\frac{E}{8\left(1-\rho^{2}\right)}\right) .
$$




$$
\mathrm{P}\left(\boldsymbol{h}_{j} \rightarrow \boldsymbol{h}_{k}\right)_{\mathrm{opt}}=Q\left(\sqrt{\frac{E}{4\left(1-\rho^{2}\right)}\left(\frac{\left\|\hat{\boldsymbol{h}}_{j}^{I}-\hat{\boldsymbol{h}}_{k}^{I}\right\|^{2}}{\delta_{I}^{2}}+\frac{\left\|\hat{\boldsymbol{h}}_{j}^{Q}-\hat{\boldsymbol{h}}_{k}^{Q}\right\|^{2}}{\delta_{Q}^{2}}-\frac{2 \rho\left(\hat{\boldsymbol{h}}_{j}^{I}-\hat{\boldsymbol{h}}_{k}^{I}\right)^{T}\left(\hat{\boldsymbol{h}}_{j}^{Q}-\hat{\boldsymbol{h}}_{k}^{Q}\right)}{\delta_{I} \delta_{Q}}\right)}\right)=Q\left(\sqrt{\frac{E \lambda_{\mathrm{opt}}}{4\left(1-\rho^{2}\right)}}\right)
$$

Asymptotic error probability (when the energy $E$ has a high value) can be derived as

$$
\overline{\mathrm{P}}\left(\boldsymbol{h}_{j} \rightarrow \boldsymbol{h}_{k}\right)_{\mathrm{opt}} \approx \frac{1}{2}\left(\frac{1}{\sqrt{\frac{E^{2}}{16\left(1-\rho^{2}\right) \delta_{I}^{2} \delta_{Q}^{2}}}}\right)^{N_{r}} .
$$

Two interesting results can be seen from (23). First, it is clear that the diversity gain equals to the number of $\mathrm{Rx}$ antennas $\left(N_{r}\right)$. Second, the results match the results when the noise at the Rx is PGN under the assumption of perfect CSI (i.e., $\rho=0, \sigma_{I}^{2}=\sigma_{Q}^{2}=\sigma_{n}^{2} / 2$ and $\sigma_{e}^{2}=0$ ). In this case, $\overline{\mathrm{P}}\left(\boldsymbol{h}_{j} \rightarrow \boldsymbol{h}_{k}\right)_{\mathrm{opt}} \approx \frac{1}{2}\left(\frac{1}{E / 2 \sigma_{n}^{2}}\right)^{N_{r}}$, hence proving to be a validation for the consequent analysis.

\section{B. PEP of Sub-Optimal ML Detector}

Using the decision rule in (11), PEP of the sub-optimal ML detector can be written under the assumption of equally likely information as follows (note that $\boldsymbol{y}=\sqrt{E} \boldsymbol{h}_{j}+\boldsymbol{n}$ )

$$
\mathrm{P}\left(\boldsymbol{h}_{j} \rightarrow \boldsymbol{h}_{k}\right)_{\text {sub }}=\operatorname{Pr}\left\{\left\|\boldsymbol{y}-\sqrt{E} \hat{\boldsymbol{h}}_{j}\right\|^{2}>\left\|\boldsymbol{y}-\sqrt{E} \hat{\boldsymbol{h}}_{k}\right\|^{2}\right\},
$$

and it can be simplified to

$$
\mathrm{P}\left(\boldsymbol{h}_{j} \rightarrow \boldsymbol{h}_{k}\right)_{\mathrm{sub}}=\operatorname{Pr}\left\{E\left\|\left(\hat{\boldsymbol{h}}_{j}-\hat{\boldsymbol{h}}_{k}\right)\right\|^{2}+D<0\right\},
$$

where $D=2 \sqrt{E} \Re\left\{\boldsymbol{n}^{T}\left(\hat{\boldsymbol{h}}_{j}-\hat{\boldsymbol{h}}_{k}\right)\right\}$ is a Gaussian RV with zero mean and variance of

$$
\begin{aligned}
\sigma_{D}^{2}=4\left\|\hat{\boldsymbol{h}}_{j}^{I}-\hat{\boldsymbol{h}}_{k}^{I}\right\|^{2} \delta_{I}^{2}+4\left\|\hat{\boldsymbol{h}}_{j}^{Q}-\hat{\boldsymbol{h}}_{k}^{Q}\right\|^{2} \delta_{Q}^{2} \\
+8 \rho\left(\hat{\boldsymbol{h}}_{j}^{I}-\hat{\boldsymbol{h}}_{k}^{I}\right)^{T}\left(\hat{\boldsymbol{h}}_{j}^{Q}-\hat{\boldsymbol{h}}_{k}^{Q}\right) \delta_{I} \delta_{Q} .
\end{aligned}
$$

Therefore, after some simplifications, $\mathrm{P}\left(\boldsymbol{h}_{j} \rightarrow \boldsymbol{h}_{k}\right)_{\text {sub }}$ can also be written as in (27) at the top of this page, where $\lambda_{s u b}=$ $\frac{E\left\|\hat{\boldsymbol{h}}_{j}-\hat{\boldsymbol{h}}_{k}\right\|^{4}}{4\left\|\hat{\boldsymbol{h}}_{j}^{I}-\hat{\boldsymbol{h}}_{k}^{I}\right\|^{2} \delta_{I}^{2}+4\left\|\hat{\boldsymbol{h}}_{j}^{Q}-\hat{\boldsymbol{h}}_{k}^{Q}\right\|^{2} \delta_{Q}^{2}+8 \rho\left(\hat{\boldsymbol{h}}_{j}^{I}-\hat{\boldsymbol{h}}_{k}^{I}\right)^{T}\left(\hat{\boldsymbol{h}}_{j}^{Q}-\hat{\boldsymbol{h}}_{k}^{Q}\right) \delta_{I} \delta_{Q}}$. However, it is not easy to find the PDF of $\lambda_{\text {sub }}$. Therefore, the APEP for the sub-optimal case is calculated by averaging the instantaneous PEP values over a large number of channel realizations.

\section{Bit Error Rate (BER)}

The BER value of the optimal and the sub-optimal detectors can be calculated by using (17) and (27), and utilizing the union bounding technique in [14] as follows

$$
P_{e, \mathrm{~b}} \leq \sum_{j=1}^{N_{t}} \sum_{\hat{j}=j+1}^{N_{t}} \frac{2 C(j, \hat{j})}{N_{t} \log _{2}\left(N_{t}\right)} \mathrm{P}\left(\boldsymbol{h}_{j} \rightarrow \boldsymbol{h}_{k}\right)_{\mathrm{b}}
$$

where $\mathrm{b} \in\{\mathrm{opt}, \mathrm{sub}\}, C(j, \hat{j})$ is the number of bits in error. Additionally, the average BER (ABER) of the optimal case can also be calculated from this expression. In this case, $\bar{P}\left(\boldsymbol{h}_{j} \rightarrow \boldsymbol{h}_{k}\right)_{\text {opt }}$ in (21) should be written instead of $\mathrm{P}\left(\boldsymbol{h}_{j} \rightarrow \boldsymbol{h}_{k}\right)_{\mathrm{b}}$ in (28).

\section{NumERICAL ANALYSIS AND RESUlts}

In this section, the impacts of IGN and ICSI on the error performance of the optimal and sub-optimal SSK MIMO Rxs are presented. The analytical derivations are validated using the computer simulations. $2 \times 2$ SSK configuration is considered in this study; however, the analysis can be generalized for any combination of $N_{t}$ and $N_{r}$.

In Fig. 1, the ABER performance of the optimal and suboptimal detectors are given in the presence of ICSI with $\sigma_{e_{I}}^{2}=\sigma_{I}^{2} / E$ and $\sigma_{e_{Q}}^{2}=\sigma_{Q}^{2} / E$ for the optimal $\mathrm{Rx}$ and $\sigma_{e_{I}}^{2}=\sigma_{n}^{2} / 2 E$ and $\sigma_{e_{Q}}^{2}=\sigma_{n}^{2} / 2 E$ for the sub-optimal one at different values of $\sigma_{I}^{2}, \sigma_{Q}^{2}$ and $\rho$ (note that $\sigma_{n}^{2}=\sigma_{I}^{2}+\sigma_{Q}^{2}=1$ ).

Two main points could be concluded from Fig. 1. First, it is clear that the proposed optimal ML detector is superior to the sub-optimal one for all cases. Second, although increasing the improperness (i.e more the difference between $\sigma_{I}^{2}$ and $\sigma_{Q}^{2}$ values or higher $\rho$ values) of the noise does not provide considerable performance enhancement for the sub-optimal detector, it noticeably increases the performance of the optimal one; for instance, approximately $4 \mathrm{~dB}$ improvement is ensured on the performance of the optimal $\mathrm{Rx}$ design for $\sigma_{I}^{2}=0.2$ and $\rho=0.7$ case when $\mathrm{ABER}=10^{-3}$. This positive effect is proved by all cases of Gaussian noise improperness: a) non-identical and uncorrelated ( i.e. $\sigma_{I}^{2} \neq \sigma_{Q}^{2}$ and $\rho=0$ ) RVs, b) identical and correlated (i.e. $\sigma_{I}^{2}=\sigma_{Q}^{2}$ and $\rho \neq 0$ ) RVs, c) non-identical and correlated RVs. It is observed that the best results are obtained for the last case.

All results of Fig. 1 are applicable to Fig. 2. In addition, as it is expected, the perfect CSI provides better results compared to Figs. 1 and 2 prove that the optimal SSK Rx outperforms the traditional sub-optimal in all cases.

Fig.s 3.a and 3.b consider the performance of the optimal $\mathrm{Rx}$ in different cases. In Fig. 3.a, the numerical results are given assuming $\rho=0$ with different values of $\sigma_{I}^{2}$ and $\sigma_{Q}^{2}$. It can be concluded that increasing the difference between the values of $\sigma_{I}^{2}$ and $\sigma_{Q}^{2}$ provides better error performance. In Fig. 3.b, the results are presented considering a fixed value of $\sigma_{I}^{2}=\sigma_{I}^{2}=0.5$ and different values of $\rho$. It is obvious that increasing the correlation factor $\rho$ enhances the performance of the Rx.

Finally, the ABER performance evaluation of the optimal ML detector under the effect of ICSI employing the CRLB variances of $\sigma_{e_{I}}^{2}$ and $\sigma_{e_{Q}}^{2}$ in (5) at different numbers of pilots $(1,3,5,7$ and 10) is presented in Fig. 4. This figure helps not only to evaluate the system performance but also predict the estimator performance. The perfect CSI is also included to the figure for comparison. It can be noticed that increasing number of pilots enhances the system performance. This estimator can work as a benchmark to find the CRLB variances of $\sigma_{e_{I}}^{2}$ and $\sigma_{e_{Q}}^{2}$ under the effect of IGN. 


$$
\mathrm{P}\left(\boldsymbol{h}_{j} \rightarrow \boldsymbol{h}_{k}\right)_{\mathrm{sub}}=Q\left(\sqrt{\frac{E\left\|\hat{\boldsymbol{h}}_{j}-\hat{\boldsymbol{h}}_{k}\right\|^{4}}{4\left\|\hat{\boldsymbol{h}}_{j}^{I}-\hat{\boldsymbol{h}}_{k}^{I}\right\|^{2} \delta_{I}^{2}+4\left\|\hat{\boldsymbol{h}}_{j}^{Q}-\hat{\boldsymbol{h}}_{k}^{Q}\right\|^{2} \delta_{Q}^{2}+8 \rho\left(\hat{\boldsymbol{h}}_{j}^{I}-\hat{\boldsymbol{h}}_{k}^{I}\right)^{T}\left(\hat{\boldsymbol{h}}_{j}^{Q}-\hat{\boldsymbol{h}}_{k}^{Q}\right) \delta_{I} \delta_{Q}}}\right)=Q\left(\sqrt{\lambda_{\mathrm{sub}}}\right)
$$

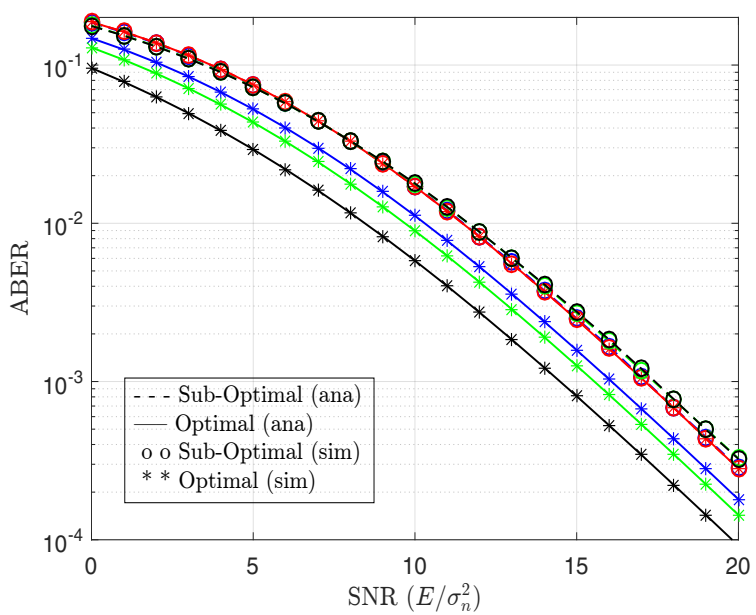

Figure 1. ABER performance of the SSK-MIMO system Rxs in the presence of ICSI and IGN: red, blue, green and black curves represent the cases of $\sigma_{I}^{2}=0.5, \rho=0 ; \sigma_{I}^{2}=0.2, \rho=0 ; \sigma_{I}^{2}=0.5, \rho=0.7$ and $\sigma_{I}^{2}=0.2, \rho=$ 0.7 , respectively.

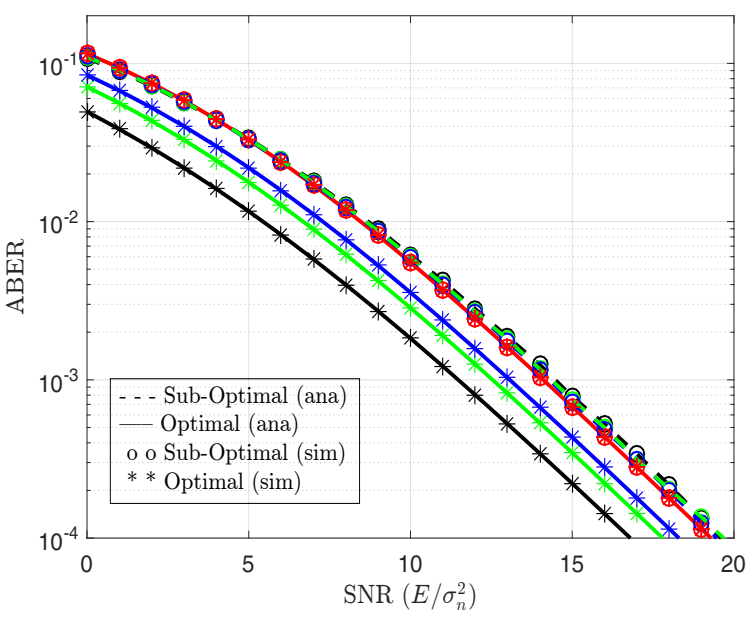

Figure 2. ABER performance of the SSK-MIMO system Rxs in the case of perfect CSI and IGN: red, blue, green and black curves represent $\sigma_{I}^{2}=$ $0.5, \rho=0 ; \sigma_{I}^{2}=0.2, \rho=0 ; \sigma_{I}^{2}=0.5, \rho=0.7$ and $\sigma_{I}^{2}=0.2, \rho=0.7$, respectively.

\section{CONCLUSION}

This work has introduced an optimal ML detector for SSK in the presence of IGN and ICSI. This optimal detector has been compared with the sub-optimal one, which applies the same decision rule that would be used in case of PGN and ICSI. Furthermore, we have presented an accurate framework for analyzing the performance of SSK over Rayleigh fading channels with an arbitrary number of Rx and in the presence of IGN and ICSI at the Rx node. Numerical and computer
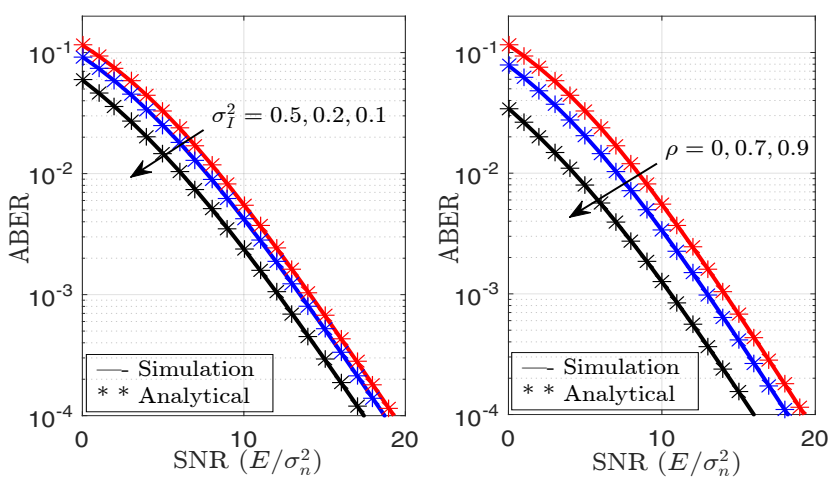

Figure 3. ABER performance of the optimal ML detector under the effect of ICSI and IGN: a) $\rho=0$, b) $\sigma_{I}^{2}=\sigma_{Q}^{2}=0.5$.

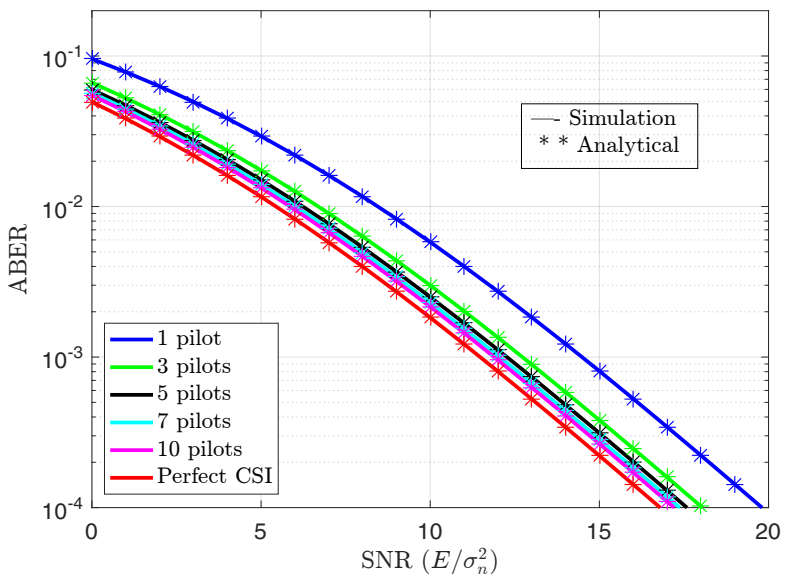

Figure 4. ABER performance of the optimal SSK MIMO Rxs under the effect of IGN using CRLB at different values of pilots $\left(N_{p}\right)$ : red, blue, green and black curves represent $\sigma_{I}^{2}=0.5, \rho=0 ; \sigma_{I}^{2}=0.2, \rho=0$; $\sigma_{I}^{2}=0.5, \rho=0.7$ and $\sigma_{I}^{2}=0.2, \rho=0.7$, respectively.

simulation results have validated the accuracy of our analytical derivations. We have shown that the performance of the optimal SSK Rx in the presence of IGN with perfect CSI and ICSI outperforms the sub-optimal one and the performance difference are noteworthy in some cases. In addition, an exact closed form Cramer-Rao bound expression is calculated for evaluating the channel estimation accuracy in the presence of IGN. This work points out the necessity of considering IGN effect for the future researches.

\section{APPENDIX}

\section{A. Proof of Lemma}

ML estimator (MLE) is used to find the CRLB. The joint 
likelihood function can be given as

$$
\begin{aligned}
& P_{\bar{y}}(\bar{y} ; \theta)=\left(\frac{1}{2 \pi \sigma_{I} \sigma_{Q} \sqrt{1-\rho^{2}}}\right)^{N_{P}} \exp \left(-\frac{1}{2\left(1-\rho^{2}\right)}\right. \\
& \sum_{N=0}^{N_{p}-1}\left[\frac{\left(y^{I}-\sqrt{E} h^{I}\right)^{2}}{\sigma_{I}^{2}}+\frac{\left(y^{Q}-\sqrt{E} h^{Q}\right)^{2}}{\sigma_{Q}^{2}}-\right. \\
&\left.\left.\frac{2 \rho\left(y^{I}-\sqrt{E} h^{I}\right)\left(y^{Q}-\sqrt{E} h^{Q}\right)}{\sigma_{I} \sigma_{Q}}\right]\right) .
\end{aligned}
$$

The log-likelihood can be written as

$$
\begin{aligned}
\ln \left(\mathrm{P}_{\overline{\mathrm{y}}}(\overline{\mathrm{y}} ; \theta)\right)= & -N_{P} \ln \left(2 \pi \sigma_{\mathrm{I}} \sigma_{\mathrm{Q}} \sqrt{1-\rho^{2}}\right)-\frac{1}{2\left(1-\rho^{2}\right)} \\
& \sum_{N=0}^{N_{p}-1}\left[\frac{\left(y^{I}-\sqrt{E} h^{I}\right)^{2}}{\sigma_{I}^{2}}+\frac{\left(y^{Q}-\sqrt{E} h^{Q}\right)^{2}}{\sigma_{Q}^{2}}-\right. \\
& \left.\frac{2 \rho\left(y^{I}-\sqrt{E} h^{I}\right)\left(y^{Q}-\sqrt{E} h^{Q}\right)}{\sigma_{I} \sigma_{Q}}\right]
\end{aligned}
$$

The elements of the matrix (3) can be found as

$$
I_{\left(h^{I}\right)}=-\mathbb{E}\left\{\frac{\partial^{2} \ln \left(\mathrm{P}_{\overline{\mathrm{y}}}(\overline{\mathrm{y}} ; \theta)\right)}{\partial h^{I^{2}}}\right\} .
$$

The first derivative of $\ln \left(\mathrm{P}_{\overline{\mathrm{y}}}(\overline{\mathrm{y}} ; \theta)\right)$ is given by

$$
\begin{aligned}
\frac{\partial \ln \left(\mathrm{P}_{\overline{\mathrm{y}}}(\overline{\mathrm{y}} ; \theta)\right)}{\partial h^{I}} & =\frac{\sqrt{E}}{\left(1-\rho^{2}\right)} \times \\
& \sum_{N=0}^{N_{p}-1}\left[\frac{\left(y^{I}-\sqrt{E} h^{I}\right)}{\sigma_{I}^{2}}-\frac{\rho\left(y^{Q}-\sqrt{E} h^{Q}\right)}{\sigma_{I} \sigma_{Q}}\right] .
\end{aligned}
$$

The second derivative of $\ln \left(\mathrm{P}_{\overline{\mathrm{y}}}(\overline{\mathrm{y}} ; \theta)\right)$ is given by

$$
\frac{\partial^{2} \ln \left(\mathrm{P}_{\overline{\mathrm{y}}}(\overline{\mathrm{y}} ; \theta)\right)}{\partial h^{I^{2}}}=-\frac{N_{p} E}{\left(1-\rho^{2}\right) \sigma_{I}^{2}} .
$$

From which (31), $I_{\left(h^{I}\right)}$ can be given by

$$
I_{\left(h^{I}\right)}=\frac{N_{p} E}{\left(1-\rho^{2}\right) \sigma_{I}^{2}} .
$$

Similar to the derivation of $I_{\left(h^{I}\right)}, I_{\left(h^{Q}\right)}$ can be derived as

$$
I_{\left(h^{Q}\right)}=\frac{N_{p} E}{\left(1-\rho^{2}\right) \sigma_{Q}^{2}} .
$$

In the same way, $I_{\left(h^{I}, h^{Q}\right)}=I_{\left(h^{Q}, h^{I}\right)}$ is obtained from

$$
I_{\left(h^{I}, h^{Q}\right)}=-\mathbb{E}\left\{\frac{\partial^{2} \ln \left(\mathrm{P}_{\overline{\mathrm{y}}}(\overline{\mathrm{y}} ; \theta)\right)}{\partial h^{I} \partial h^{Q}}\right\}=-\frac{N_{p} \rho E}{\left(1-\rho^{2}\right) \sigma_{I} \sigma_{Q}} .
$$

Combining (34), (35), and (36) leads to (4), which concludes the proof.

\section{ACKNOWLEDGMENT}

This work was supported in part by a Discovery Grant (DG) from the Natural Sciences and Engineering Research Council (NSERC) of Canada and Research Development Fund (RDF) from Lakehead University. This work was also supported in part by The Scientific and Technological Research Council of Turkey (TUBITAK) BIDEB-2214 International Doctoral Research Fellowship Programme. E. Basar acknowledges the support of Turkish Academy of Sciences Outstanding Young Scientists Award Programme.

\section{REFERENCES}

[1] E. Dahlman et al., "5G radio access," Ericsson Review, vol. 6, pp. 2-7, 2014.

[2] E. Basar et al., "Index modulation techniques for next-generation wireless networks," IEEE Access, vol. 5, pp. 16693-16746, 2017.

[3] Y. A. Chau and S.-H. Yu, "Space modulation on wireless fading channels," in Proc. 2011 IEEE 54th Veh. Technol. Conf. (VTC), vol. 3, 2001, pp. 1668-1671 vol.3.

[4] J. Jeganathan, A. Ghrayeb, L. Szczecinski, and A. Ceron, "Space shift keying modulation for MIMO channels," IEEE Trans. Wireless Commun., vol. 8, no. 7, pp. 3692-3703, July 2009.

[5] C. Xu et al., "Two decades of MIMO design tradeoffs and reducedcomplexity mimo detection in near-capacity systems," IEEE Access, vol. 5, pp. 18564-18 632, 2017.

[6] S. S. Ikki and R. Mesleh, "A general framework for performance analysis of space shift keying (SSK) modulation in the presence of gaussian imperfect estimations," IEEE Commun. Letters, vol. 16, no. 2, pp. 228-230, Feb. 2012.

[7] F. S. Al-Qahtani, S. Ikki, M. D. Renzo, and H. Alnuweiri, "Performance analysis of space shift keying modulation with imperfect estimation in the presence of co-channel interference," IEEE Commun. Letters, vol. 18, no. 9, pp. 1587-1590, Sep. 2014.

[8] A. Afana and S. Ikki, "Analytical framework for space shift keying MIMO systems with hardware impairments and co-channel interference," IEEE Commun. Letters, vol. 21, no. 3, pp. 488-491, March 2017.

[9] S. Javed, O. Amin, S. S. Ikki, and M. S. Alouini, "Impact of improper gaussian signaling on hardware impaired systems," in 2017 IEEE Int. Conf. Commun. (ICC), May 2017, pp. 1-6.

[10] J. M. Romero-Jerez and F. J. Lopez-Martinez, "On the distribution of the squared norm of non-circular complex Gaussian random variables with applications," in Proc. 2015 IEEE Int.Symp. on Inf. Theory (ISIT), June 2015, pp. 341-345.

[11] A. S. Aghaei, K. N. Plataniotis, and S. Pasupathy, "Maximum likelihood binary detection in improper complex gaussian noise," in 2008 IEEE Int. Conf. Acoustics, Speech and Signal Processing, March 2008, pp. 3209-3212.

[12] R. Mesleh and S. S. Ikki, "On the effect of Gaussian imperfect channel estimations on the performance of space modulation techniques," in 2012 IEEE 75th Veh. Technol. Conf. (VTC Spring), May 2012, pp. $1-5$.

[13] A. Afana, N. Abu-Ali, and S. Ikki, "On the joint impact of hardware and channel imperfections on cognitive spatial modulation MIMO systems: Cramer-rao bound approach," IEEE Systems J., pp. 1-12, 2018.

[14] F. S. Al-Qahtani, Y. Huang, M. D. Renzo, S. Ikki, and H. Alnuweiri, "Space shift keying MIMO system under spectrum sharing environments in rayleigh fading," IEEE Commun. Letters, vol. 18, no. 9, pp. 1503-1506, Sep. 2014. 